\title{
Prevenção à cegueira em crianças de 3 a 6 anos assistidas pelo programa de saúde da família (PSF) do Morro do Alemão - Rio de Janeiro
}

\section{Blindness prevention on 3 to 6 years old children at a health family program assisted community in Morro do Alemão - Rio de Janeiro}

Giancarlo Cardoso Jeveaux, Arlindo José Freire Portes², Abelardo de Souza Couto Júnior; Flavio Shinzato ${ }^{4}$

\begin{tabular}{l}
\hline RESUMO \\
Objetivo: Este trabalho visou fazer um levantamento da prevalência de ambliopia e \\
fatores ambliopiogênicos em crianças com idade pré-escolar em áreas assistidas pelo \\
Programa de Saúde da Família no Morro do Alemão-RJ. Métodos: Crianças com idade \\
entre 3 e 6 anos foram submetidas a exame de triagem visual. Os resultados positivos \\
foram confirmados por exame oftalmológico realizado sob cicloplegia na própria uni- \\
dade de saúde. Foram aplicados questionários semi-estruturados aos acompanhantes \\
das crianças e aos membros de todas as equipes de saúde da unidade. Resultados: De \\
559 crianças convocadas para triagem,265 (47,4\%) compareceram e, destas, 127 (48\%) \\
foram encaminhadas para exame oftalmológico completo. Tiveram o exame ocular \\
normal 138 (52\%) das crianças examinadas pela triagem. Compareceram para exame \\
especializado 81 (63,7\%) crianças. Destas, 9 (4,1\%) crianças tiveram diagnóstico de \\
ambliopia. Quatro (1,8\%) por estrabismo, uma (0,46\%) por privação de estímulo, uma \\
(0,46\%) por anisometropia e três (33,3\%) por erros refracionais isometrópicos. Conclu- \\
são: Os dados obtidos pela triagem seguida de exame ocular especializado executada \\
na unidade de atenção primária à saúde (PSF) do Morro do Alemão-RJ, mostraram que \\
os exames de triagem realizados são relevantes para a detecção de ambliopia e fatores \\
ambliopiogênicos e para a promoção da saúde ocular infantil.
\end{tabular}

Descritores: Ambliopia/epidemiologia; fatores ambliopiogênicos, Programa saúde da família; Cegueira; Criança; Pré-escolar; Saúde escolar

\footnotetext{
'Professor de Medicina da Universidade de Vila Velha; Médico Oftalmologista da Santa Casa de Misericórdia de Vitória; Médico Oftalmologista do Vitória Apart Hospital - Vitória (ES), Brasil;

Professor adjunto de Oftalmologia da Universidade Estácio de Sá - Rio de Janeiro (RJ), Brasil;

${ }^{3}$ Professor adjunto de Oftalmologia da Universidade de Valença - Rio de Janeiro (RJ), Brasil;

${ }^{4}$ Pos-graduando de Oftalmologia do Hospital Geral de Bonsucesso - Rio de Janeiro (RJ), Brasil;

Trabalho realizado como tese de mestrado em Saúde da Família da Universidade Estácio de Sá - Rio de Janeiro (RJ), Brasil.
}

Recebido para publicação em: 27/5/2008 - Aceito para publicação em 1/9/2008 


\section{INTRODUÇÃO}

$\mathbf{N}$ o Brasil, existem 98 milhões de pessoas comalgum tipo de deficiência visual, dos quais 80 milhões não têm acesso a qualquer tratamento ${ }^{(1-4)}$. Em relação à atenção a saúde ocular infantil, não há programa público de prevenção à cegueira ou deficiência visual em crianças com idade pré-escolar a partir de 3 anos. Contudo, ocorrem campanhas de prevenção que visam atender às crianças na idade escolar, que envolvem na sua maioria, oftalmologistas e professoras primárias. A campanha "Olho no Olho" constitui exemplo de parceria entre o Conselho Brasileiro de Oftalmologia, o Ministério da Educação e Secretarias de Educação e Saúde - estaduais e municipais - que uniram esforços para detecção e tratamento de distúrbios visuais de escolares. Foram 3 milhões e 100 mil triagens visuais em uma campanha que atingiu crianças de 7 anos ou mais, portanto, não sendo efetiva em eliminar várias causas de cegueira infantil que ocorrem mais precocemente e de maior freqüência ${ }^{(5-7)}$.

A ambliopia é a principal causa de cegueira infantil monocular e deficiência da visão infantil, devendo ser revertida antes do desenvolvimento pleno do sistema visual, o qual ocorre por volta dos 6 a 7 anos ${ }^{(3,5,8-10)}$. Como após essa idade, o problema torna-se irreversível, constitui uma questão de saúde pública, envolvendo todos seus aspectos sociais e econômicos. Acredita-se que a falta de um programa público que incorpore a prática de medidas de prevenção à cegueira e deficiência visual no Brasil, na rotina das equipes de saúde, especialmente nesta faixa etária de grande maleabilidade na formação do sistema visual, contribua para sua alta prevalência atual.

Considerando ser de pouca praticidade a realização de exame oftalmológico especializado em cada criança em idade pré-escolar, métodos de triagem visual executados por outros membros das equipes de saúde, permanecem como a forma mais viável e acessível de detectar crianças com ambliopia, para as quais a intervenção precoce ou o tratamento oclusivo é essencial para o sucesso na recuperação visual.

\section{Métodos}

Durante um período de 1 ano e meio, as crianças foram avaliadas semanalmente e em dia pré-estabelecido e acordado com a coordenação da unidade e suas equipes de saúde, por um profissional qualificado após consentimento dos pais ou responsáveis.
Foi realizada a seguinte avaliação para a triagem das crianças: anamnese dirigida, exame ocular ectoscópico, medida da acuidade visual com tabela de Snellen a 6 metros, teste de Estereopsia (Teste de Titmus), exame do reflexo vermelho com oftalmoscopia direta e teste de cobertura monocular. Foram considerados resultados indicativos de encaminhamento para avaliação ocular especializada oftalmológica, a fim de diagnosticar patologias que causem cegueira ou deficiência visual: queixa de astenopia, qualquer alteração ectoscópica que possa causar privação visual, visão menor ou igual a $0,7 \mathrm{ou}$ 20/30 para crianças entre 4 e 6 anos e menor ou igual a 0,5 ou $20 / 40$ para crianças de 3 anos, a presença de opacidade dos meios ou desvio ocular, ausência de visão estereoscópica.

Inicialmente, a avaliação ocular especializada foi realizada no Hospital Geral de Bonsucesso, com agendamento do paciente, com hora e data previamente marcada e incluindo: medida da acuidade visual a 6 metros com tabela de Snellen, refração objetiva sob cicloplegia e refração subjetiva, quando a criança colaborava, avaliação da motilidade ocular, biomicroscopia, oftalmoscopia indireta sob midríase e tonometria quando necessário.

Os critérios diagnósticos para as afecções oculares encontradas neste trabalho foram:

Ametropia - Qualquer erro refracional maior ou igual a $0,5 \mathrm{D}$;

Ambliopia - Déficit funcional com a melhor correção óptica de acuidade visual sem causa orgânica, levando a uma diferença de pelo menos duas linhas na tabela de Snellen, quando se comparam os olhos. Consideram-se as formas anisometrópicas, estrábicas, refracionais e de privação;

Estrabismo - Ausência de alinhamento do eixo ocular manifesto;

Anisometropia - Diferença de duas ou mais dioptrias, tanto esférica como cilíndrica, entre a refração dos dois olhos. tionários:

Realização de entrevistas com aplicação de ques-

Foram entrevistados pelo pesquisador responsável de forma individual e isoladamente, pais ou responsáveis pelas crianças, médicos e enfermeiros da unidade de saúde do Morro do Alemão, com um questionário semi-estruturado para cada grupo (pais ou responsáveis/médicos e enfermeiros).

O objetivo do questionário era de auferir a capacidade de reconhecimento de sintomas de baixa de visão ou de necessidade de uso de lentes corretivas no ambien- 
te familiar e/ou doméstico, o nível de conhecimento sobre as principais e mais comuns patologias oculares que acometem crianças na idade pré-escolar no Brasil.

\section{Resultados}

Do total de 740 pacientes de 3 a 6 anos, cobertos pelas 5 equipes de saúde que compõem o PSF do Morro do Alemão, foram convidadas para avaliação 559 (75,5\%) crianças de 3 a 6 anos, as quais fazem parte da área de abrangência de 3 das equipes de saúde da unidade do Morro do Alemão (equipes 1, 4 e 5). As duas equipes não avaliadas (equipes 2 e 3), respondem por $181(24,5 \%)$ crianças da faixa etária de interesse para a pesquisa. Foram excluídas do trabalho, todas as crianças que não compareceram a triagem e as que fizeram os exames de triagem, mas não compareceram ao exame oftalmológico completo.

Nos resultados do estudo de 559 crianças entre 3 e 6 anos de idade assistidas pelo Programa de Saúde de Família da comunidade do Morro do Alemão (RJ) e convidadas para a triagem, $265(47,4 \%)$ foram avaliadas pela triagem visual, enquanto 294 (52,6\%) não compareceram. Foram excluídas do estudo as 294 (52,6\% ) crianças que não compareceram à triagem visual em conjunto com as $46(36,2 \%)$ crianças que não compareceram à realização do exame especializado.

Do total das 265 crianças que compareceram à triagem, 138 (52\%) não apresentaram alterações oculares e $127(48 \%)$ foram encaminhadas para exame sob cicloplegia por apresentarem alguma alteração aos exames ou por não colaborarem (Gráfico 1).

Os motivos que levaram ao encaminhamento para ciclopegia se distribuíram de acordo com o gráfico abaixo (Gráfico 1).

Dos 127 pacientes encaminhados, $81(63,7 \%)$ compareceram para exame especializado e, destes, 36 (44,4\%) apresentaram erros refracionais, sendo necessário a prescrição de lentes corretoras. Dentro do total das 36 crianças que apresentaram algum tipo de ametropia, quatro (5\%)apresentaram estrabismo e uma (1,2\%) apresentou ptose congênita unilateral da pálpebra superior do olho esquerdo. Nesses casos também foram constatados erros refracionais associados. Das quatro crianças diagnosticadas com estrabismo, uma $(25 \%)$ apresentou exotropia intermitente e as três (75\%) restantes apresentaram esotropia, sendo todas, inicialmente, tratadas com terapia oclusiva e utilização de lentes corretivas para correção dos erros de refração associados.

Do total das crianças selecionadas para exame completo, 48 (38\%) tinham 3 anos, 34 (27\%) tinham 4 anos, 22 (17\%) tinham 5 anos e 23 (18\%) tinham a idade de 6 anos.

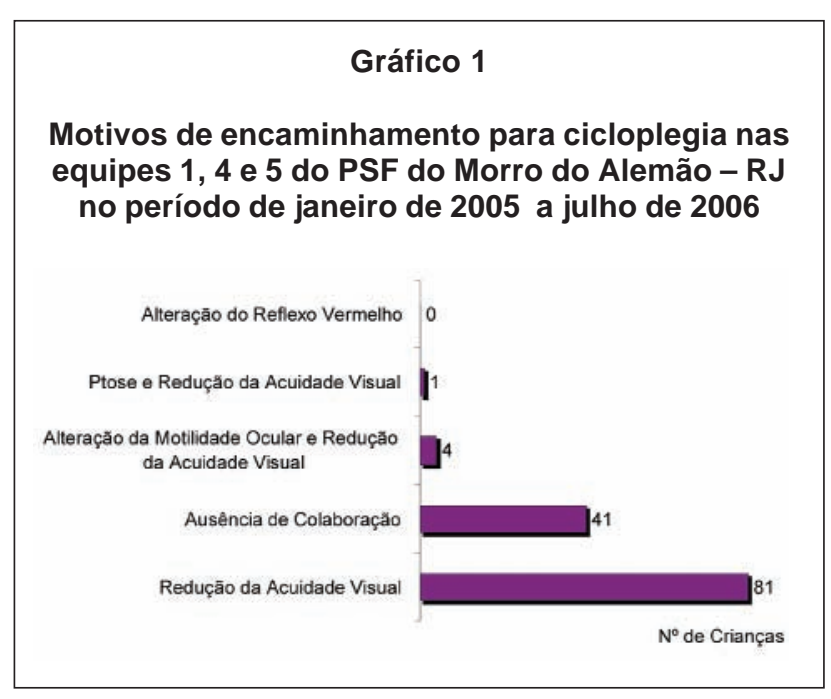

Os diagnósticos, após exame especializado, dos tipos, freqüência e prevalência de ametropias das 81 crianças da amostra se comportaram da seguinte maneira: 17 (7,8\%) com hipermetropia, 7 (3,2\%) com miopia, 4 $(1,8 \%)$ com astigmatismo simples, $4(1,8 \%)$ com astigmatismo hipermetrópico composto e $3(1,4 \%)$ com astigmatismo miópico composto.

A prevalência de doenças oftalmológicas nas crianças com idade de 3 a 6 anos de idade, no período de janeiro de 2005 a julho de 2006, quando foi realizada a pesquisa, foi da ordem de $15,98 \%$, com uma margem de erro de $4,12 \%$, no nível de confiança de $95 \%$.

A tabela 1 mostra, resumidamente, os diagnósticos de ametropias após realização do exame com o especialista, sua prevalência e margem de erro calculada, com intervalo de confiança de $95 \%$.

Das 127 crianças encaminhadas para o exame especializado, $9(4,11 \%)$ tiveram diagnóstico de ambliopia. Dessas, as causas gerais de ambliopia ao final da pesquisa se dividiram em: $4(1,83 \%)$ por estrabismo, $1(0,46 \%)$ por privação de estímulo, $1(0,46 \%)$ refracional por anisometropia e $3(1,37 \%)$ refracionais isometrópicos. Dos pacientes estrábicos, 3 (75\%) apresentaram componente refracional anisometrópico associado e $1(25 \%)$ isometrópico.

Para a ambliopia, a prevalência foi da ordem de $4,11 \%$, com margem de erro de $2,21 \%$, no nível de $95 \%$ de confiança.

\section{Discussão}

Conforme Temporini e Kara-José, programas de saúde pública em oftalmologia devem visar, a promoção de saúde ocular, a organização de assistência 
Tabela 1

Diagnóstico de ametropias após exame especializado, prevalência e margem de erro nas equipes 1, 4 e 5 do PSF do Morro do Alemão - Rio de Janeiro, no período de janeiro de 2005 a julho de 2006

\begin{tabular}{lccc}
\hline Diagnóstico após exame especializado & $\mathbf{N}^{\mathbf{0}}$ de Crianças & Prevalência geral & Margem de erro \\
\hline Hipermetropia & 17 & $7.76 \%$ & $3.0 \%$ \\
Miopia & 7 & $3.20 \%$ & $2.0 \%$ \\
Astigmatismo Simples & 4 & $1.83 \%$ & $1.5 \%$ \\
Astigmatismo Hipermetrópico Composto & 4 & $1.83 \%$ & $1.5 \%$ \\
Astigmatismo Miópico Composto & 3 & $1.37 \%$ & $1.3 \%$ \\
Astigmatismo Hipermetrópico Simples & 0 & $0.00 \%$ & $0.0 \%$ \\
Astigmatismo Miópico Simples & 0 & $0.00 \%$ & $0.0 \%$ \\
Astigmatismo Misto & 0 & $0.00 \%$ & $0.0 \%$ \\
Total & 35 & $15.98 \%$ & $4.1 \%$ \\
\hline
\end{tabular}

oftalmológica e a reabilitação de deficientes visuais ${ }^{(3,11)}$. A Organização Mundial de Saúde (OMS) vem demonstrando preocupação com intervenções relativas a formação de programas de prevenção da cegueira ${ }^{(6,11)}$.

A OMS propôs o programa "VISION 2020: The Right to Sight" ${ }^{(8)}$, cujo objetivo é o de eliminar a cegueira previnível ao redor do ano $2020^{(11)}$, através da elaboração de planos de atuação junto à população, através da associação com organizações governamentais e nãogovernamentais.

Resnikoff e Pararajasegaram ainda afirmam que a assistência oftalmológica deveria estar integrada ao nível primário de atenção à saúde, desenvolvendo atividades de prevenção e o controle específico de doenças oculares, o desenvolvimento de recursos humanos, de infra-estrutura e de tecnologia apropriada ${ }^{(11)}$.

O Programa de Saúde da Família considera como objetivo ampliar o acesso da população aos serviços básicos de saúde, tendo as equipes de Saúde da Família como eixo estrutural. Considerando que:

1 - A falta de acesso ao cuidado especializado oftalmológico no Brasil tem sido atribuída à insuficiência de pessoal preparado, à distância a percorrer para obter assistência oftalmológica, especialmente em zonas rurais, ao custo do tratamento, ao desconhecimento da possibilidade de recuperação visual, ao medo dos hospitais, de médicos e da própria cirurgia ocular ${ }^{(5,10)}$;

2 - Que o Programa de Saúde da Família abrange grande parte da população no interior do Brasil e está ampliando para atingir áreas dos grandes centros urbanos;

3 - Que não há programa público para a prevenção de cegueira em crianças em idade pré-escolar;

4 - Que a ambliopia é a principal causa de cegueira infantil, que para seu diagnóstico e prevenção é ne- cessário a realização de exame ocular ${ }^{7}$, e considerando que a visão é o sentido fundamental e de maior necessidade para o pleno desenvolvimento e desempenho das atividades na sociedade;

Tendo em vista a freqüência do acometimento visual infantil pela ambliopia, a gravidade da doença, com a perda irreversível da visão após o amadurecimento do sistema visual, a simplicidade da realização do diagnóstico por métodos de triagem e as graves repercussões emocionais, familiares e sociais, para o desenvolvimento da criança amblíope e para a inclusão do adulto com deficiência visual ou cegueira na sociedade, acredita-se ser necessária a criação de um programa de triagem oftalmológica, que seja incorporado pelos profissionais de saúde que atuam em unidades de PSF, como uma rotina no desempenho das suas atividades de promoção à saúde e prevenção de doenças, no atendimento junto as crianças em idade pré-escolar, que vise a detecção precoce de doenças oculares, entre elas as ambliopizantes.

\section{ConClusÃo}

A triagem seguida de exame ocular especializado executada em unidades de atenção primária à saúde (PSF), se mostrou relevante para a detecção de ambliopia e fatores ambliopiogênicos. As informações sobre a importância do exame oftalmológico precoce para o diagnóstico e tratamento das patologias oculares de maior prevalência, causadoras de deficiência visual na infância, bem como o seu devido reconhecimento clínico, são desconhecidas tanto para leigos quanto para os profissionais da área de saúde, mostrando a necessidade de uma maior divulgação a seu respeito, assim como a da realização de treinamento básico na triagem des- 
sas doenças, junto aos médicos e enfermeiras que atuam na atenção primária em saúde, em unidades do programa de saúde da família, o que mostrou ser, além de necessário, desejo dos responsáveis pelas crianças e dos profissionais entrevistados.

Uma vez que todos os entrevistados, médicos e enfermeiros ratificaram e admitiram que as ações de prevenção à cegueira infantil, através dos procedimentos de triagem propostos, são de competência da Atenção Primária, e que eles são capazes de interferir positivamente no processo, promovendo sua devida condução para a solução adequada, concluiu-se haver a necessidade de:

1.Aquisição de instrumental adequado, associado ao planejamento logístico redirecionado para as equipes de saúde;

2. Capacitação profissional dos médicos e enfermeiros que integram as equipes de saúde;

3. Treinamento, atualização e consultoria com profissional de oftalmologia que passe a preencher o quadro de referência do GAT;

4. Referência especializada, para os casos identificados como de risco para o desenvolvimento de deficiência visual.

\section{Abstract}

Purpose: The study objective is to determine the prevalence of amblyopia and amblyopiogenic factors in children from 3 to 6 years old at a health family program assisted community in Morro do Alemão-RJ. Methods: A preschool children ocular evaluation cross sectional study will be submitted to an ophthalmic screening exam. The positive results were confirmed by oftalmologic examination under cicloplegia inside of the health centre. Were applied a semi-structure questionnaire to the health members of the health centre team and to all children accompanist Results: Five hundred fifty nine patients were convocated to the examination, but only 265 (47,4\%) were presented. $138(52 \%)$ had normal ocular exam. Were sent to complete ocular evaluation 127 (48\%) patient. 81 $(63,7 \%)$ children show up for the specialized ocular examination and that's one $36(44,4 \%)$ children showed some kind of refraction error, 4 (5\%) with strabismus and $1(1,2 \%)$ with unilateral congenital ptose. Nine (7\%) showed amblyopia diagnosis. 4 (44,4\%) for strabismus, 1 $(11,1 \%)$ for stimulus deprivation, $1(11,1 \%)$ refractional for anisometropia and $3(33,3 \%)$ refractional isometrópico. Conclusion: The results obtained of the trial examination made before the specialized ocular examination realized in the health family program in Morro do Alemão-RJ, show us that the trial exams are relevant to detect amblyopia and amblyopiogenic factors and to promote children ocular health.

Keywords: Amblyopia/epidemiology; Family health program; Blindness; Child; Child, preschool; School health

\section{REFERÊNCIAS}

1. Oliveira PR. Causas de cegueira na infância. Ara Bras Oftalmol. 1992; 55(4):172-5.

2. Couto Júnior AS, Pinto GR, Oliveira DA, Holzmeister D, Portes ALF, Neurauter R, Portes AJF. Prevalência das ametropias e oftalmopatias em crianças pré-escolares e escolares em favelas do Alto da Boa Vista, Rio de Janeiro, Brasil. Rev Bras Oftalmol. 2007; 66(5):304-8.

3. Temporini ER, Kara-José N. Níveis de prevenção de problemas oftalmológicos: propostas de investigação. Arq Bras Oftalmol. 1995; 58(3):189-92.

4. Costa DS, Klein RCA, Leite CA, Ginguerra MAA, Polati M. Ambliopia por estrabismo: estudo retrospectivo de pacientes em hospital universitário. Arq Bras Oftalmol. 2006; 69(2):181-5.

5. Conselho Brasileiro de Oftalmologia. Campanha Nacional de Reabilitação Visual - Olho no Olho [Internet]. [citado 2007 Abr 10]. Disponível em: <http://www.cbo.com.br/cbo/ campanhas_sociais.htm>. Acessado em: 10/04/2007

6. Potter AR. Preventing blindness worldwide. BMJ. 1994; 309(6956):682-3.

7. Neurauter R, Bellini AT, Couto-Júnior AS. Saúde ocular de pré-escolares e escolares dos bairros de Copacabana e Urca - Rio de Janeiro. Rev Bras Oftalmol. 2001; 60(3):199-203.

8. National prevention of blindness programmes and vision 2020. Community Eye Health. 2000; 13(36):49-50.

9. Bicas HEA, Alves AA, Uras R. Refratometria ocular. Rio de Janeiro: Cultura Médica; 2005.

10. Enzenauer RW. The efficacy of photoscreening for amblyopiagenic factors in a high risk population. Binocul Vis Strabismus Q. 2003; 18(4):233-40.

11. Resnikoff S, Pararajasegaram R. Blindness prevention programmes: past, present, and future. Bull World Health Organ. 2001; 79(3):222-6. 